\title{
The Role of Biomedical Knowledge in Clinical Reasoning: A Lexical Decision Study
}

Remy M. J. P. Rikers, MSc, PhD, Sofie Loyens, MSc, Wilco te Winkel, MSc, Henk G. Schmidt, MSc, PhD, and Patrick H. M. Sins, MSc

Abstract

\section{Purpose}

To investigate the role of biomedical and diagnostic inferences in clinical reasoning of advanced medical students and experienced family physicians using a lexical decision task.

\section{Method}

In 2002, 15 family physicians and 20 fourth-year medical students at Maastricht University medical school in The Netherlands were instructed to carefully study 60 short clinical texts consisting of signs and symptoms associated with a particular disease.
Participants read the texts on a computer screen and responded using a computer keyboard. Each text was followed by a target item (i.e., biomedical item, diagnostic item, or a nonword). Participants had to decide as quickly and accurately as possible whether the presented target item was a word or a nonword. For both groups, mean response time and mean error rate for all levels of item type were analyzed.

\section{Results}

Findings indicate that both physicians and medical students judged diagnostic target items faster and more accurately than biomedical target items. However, physicians were considerably faster than were students on judging biomedical and diagnostic target items.

\section{Conclusions}

These findings are largely in line with knowledge encapsulation in that biomedical knowledge still plays a prominent role in the physician's clinical reasoning.

Acad Med. 2005; 80:945-949 $\mathrm{n}$ an experimental procedure traditionally applied in medical expertise research, participants of different levels of medical expertise are asked to study a description of a clinical case (i.e., a text describing a patient's history, complaints, results from physical examination, and some additional findings such as laboratory data). After the participants have read the text, they are asked to provide a diagnosis and to write down everything they remembered from the text (i.e., a free-recall task). In addition, participants are asked to provide an explanation of the displayed signs and

\section{Dr. Rikers is director, Higher Education Research Centre Rotterdam, and associate professor, Department of Psychology, Erasmus University Rotterdam, The Netherlands.}

Ms. Loyens is research assistant, Department of Psychology, Erasmus University Rotterdam, The Netherlands.

Mr. te Winkel is assistant professor, Department of Psychology, Erasmus University Rotterdam, The Netherlands.

Dr. Schmidt is professor, Department of Psychology, Erasmus University Rotterdam, The Netherlands.

Mr. Sins is research assistant, The Graduate School of Teaching and Learning, University of Amsterdam, The Netherlands.

Correspondence should be addressed to Dr. Rikers, Erasmus University Rotterdam, Department of Psychology/Woudestein, P.O. Box 1738, 3000 DR Rotterdam, The Netherlands; telephone: $++31(0) 10$ 4089655; e-mail: 〈rikers@fsw.eur.nl〉. symptoms in terms of their underlying pathophysiological processes (the pathophysiological explanation). In this procedure, physicians show significantly higher diagnostic performance than do participants of lower expertise levels. ${ }^{1-7}$ The influence of expertise level on recall and pathophysiological explanations, however, has been somewhat less straightforward. Rather than a monotonically increasing function of recall with increasing medical expertise, an inverted U-shaped relation was found. That is, advanced medical students remembered more about the description and provided more detailed pathophysiological explanations than did both novices and experienced physicians. This phenomenon has become known in clinical case studies as the "intermediate effect." $6-8$

According to Schmidt and Boshuizen, ${ }^{6}$ physicians' recall and pathophysiological protocols are less elaborate because they generate many inferences that encapsulate or summarize the essential information in a clinical case. 6,7 That is, as a result of extensive and repeated encounters with the manifestations of a particular disease (e.g., through encounters with real patients), signs and symptoms have become encapsulated under clinically relevant concepts (e.g., diagnosis). This restructuring, which took place during their course of expertise development, led to abbreviations in their lines of reasoning. ${ }^{9-11}$

In order to illustrate how physicians process clinical information, consider the following text: A 65-year old man has moderate fever, precordial pain and tenderness, dry cough, dyspnea, and palpitation. His pulse is first rapid and forcible, then weak and irregular. Auscultation reveals a to and fro friction sound heard over the fourth left intercostals space near the sternum. Palpation reveals a diffuse apex beat.

An experienced physician will probably process this text as a condition called pericarditis. This is an (diagnostic) inference that summarizes or encapsulates the provided information. That is, the physician processes this set of signs and symptoms as an integrated whole.

A medical student, on the other hand, has to resort to his or her biomedical knowledge in order to explain the described signs and symptoms and to find a meaningful relationship between them. Biomedical knowledge pertains to the processes underlying the manifestations of a disease, and it incorporates knowledge about domains such as biochemistry, microbiology, and physiology. ${ }^{1,12}$ Students have acquired, through individual study and lectures, an 
extensive body of biomedical knowledge, but they have little or no knowledge about the manifestations of diseases in real patients (i.e., clinical knowledge). Their clinical reasoning process consists therefore of chains of small steps based on often detailed biomedical concepts. ${ }^{3-7}$

It is important to note that previous studies have shown that a physician's biomedical knowledge is still accessible if it is needed. $6,7,8,13,14$ For instance, Schmidt and Boshuizen ${ }^{6}$ asked participants of different levels of expertise to use their biomedical knowledge by reporting everything they knew about the pathophysiology of a disease called endocarditis. What they found was a linear relation between expertise level and propositions recalled. That is, the experienced physicians in their study knew more about the pathophysiology of endocarditis than did advanced and less advanced medical students.

Although previous studies have demonstrated that biomedical knowledge is still accessible if explicitly required, it is still unclear if this type of knowledge is actually used by an experienced physician during clinical reasoning. This issue is especially pertinent because it is an important assumption of knowledge encapsulation that biomedical knowledge is still used during clinical reasoning (i.e., if biomedical knowledge is encapsulated, it should become activated).

Furthermore, most previous studies mainly used a post hoc methodology (i.e., free recall and pathophysiological explanations) and can therefore not answer the question whether the physician's biomedical knowledge has become activated while processing the clinical information. That is, these studies cannot exclude the possibility that some of the inferences that were generated during case processing may not appear in the protocols. ${ }^{15,16}$ Therefore, physicians may have used their elaborate biomedical knowledge during clinical reasoning, but are not as inclined as are medical students to report it because they consider it of less importance. ${ }^{3}$

In order to disentangle this issue, in this study we diverged from the traditional clinical case approach and investigated the role of biomedical inferences and diagnostic inferences in the case representations of physicians and advanced medical students using a lexical decision task. We instructed experienced family physicians and advanced medical students to study a short text consisting of signs and symptoms associated with a particular disease for a short period of time. Immediately after each text a target item appeared on the computer screen. Targets could be words (i.e., biomedical inferences or diagnostic inferences based on the text) or nonwords. Participants had to decide as quickly and as accurately as possible whether a target item was a word or a nonword (i.e., lexical decision).

We hypothesized that if participants generated biomedical or diagnostic inferences in their clinical reasoning, and if one of these inferences was identical to a target item, they would be faster and more accurate in their decisions. Based on knowledge encapsulation, we predicted that physicians would be faster than advanced medical students in judging the biomedical items because they have more and better integrated biomedical knowledge. ${ }^{3-8}$ Furthermore, as a result of their large clinical experience, physicians easily recognize the pattern of signs and symptoms and hence infer the associated diagnosis faster. Consequently, physicians are also faster in their decisions about the diagnostic targets than are medical students. ${ }^{1-8}$

\section{Method}

\section{Participants}

We selected a random sample of 15 family physicians and 20 fourth-year medical students at Maastricht University medical school in The Netherlands in 2002. All physicians were practitioners with at least an MD degree and board certification and had at least seven years of clinical experience. Their ages ranged from 38 to 56 years $($ mean $=44.53, \mathrm{SD}=5.71)$. The fourth-year students were all preclinical students and had therefore no, or only very little, experience in hospitals. Their knowledge of diseases was therefore predominantly from textbooks and lectures. The students' ages ranged from 22 to 26 years $($ mean $=23.07, \mathrm{SD}=1.22$ ). Students and physicians received a financial compensation for their participation.

\section{Materials}

The materials consisted of 60 short descriptions of signs and symptoms associated with a particular disease commonly seen by a family physician. With the help of two family physicians, we adapted all texts from a medical handbook. ${ }^{17}$ The length of each description, in terms of number of words, was about the same $($ mean $=59, \mathrm{SD}=$ 3.5). In contrast to typical clinical cases, which describe real patient data, these texts were more theoretical and contained no ambiguous or conflicting information. For example, the text associated with a myocardial infarction was:

\begin{abstract}
Prolonged heavy pressure or squeezing pain in the center of the chest behind the sternum. The pain may spread to the shoulder, neck, arm, and fourth and fifth fingers of the left hand; to the back, to the teeth, on to the jaw. These symptoms may be accompanied by nausea and vomiting, sweating, and shortness of breath.
\end{abstract}

In order to determine potential biomedical and diagnostic inferences, we used the "three-pronged" approach advocated by Magliano and Graesser. ${ }^{18,19}$ This approach has been advocated for studies on inference generation because inferences are not directly manifested in the text, so it is essential to use a method to expose inferences and to test whether they are generated during normal reading. It involves three procedural criteria:

- theoretical predications in order to identify a priori text-based inferences (e.g., knowledge encapsulation predicts that physicians easily generate biomedical inferences because signs and symptoms trigger their underlying biomedical knowledge);

- collecting verbal protocol data to reveal inferences that a group of readers make as they comprehend the text; and

- using behavioral measures to test and validate the first two procedures (e.g., lexical decision and naming latencies of test words, fixation times on words during eye tracking). ${ }^{15,16}$

Following the three-pronged approach, we first adopted the knowledge encapsulation view of Schmidt and colleagues $^{6-8}$ as our discourse model to identify what classes of inferences are expected to be generated. Second, in order to identify specific inferences that surface to consciousness, we asked ten medical students and ten family physicians, none of whom participated in the final experiment, to think aloud and express whatever came to their mind while processing our short descriptions of 
Table 1

Participants' Mean Response Time in Milliseconds and Standard Errors, by Expertise Level and Item Type, from a Study on the Role of Biomedical Knowledge in Clinical Reasoning, Maastricht University Medical School, The Netherlands, 2002

\begin{tabular}{lrrr} 
& \multicolumn{2}{c}{ Mean $(\mathrm{SE})$ response time } \\
\cline { 2 - 4 } Participant's expertise level & $\begin{array}{r}\text { Biomedical } \\
\text { Items }\end{array}$ & $\begin{array}{r}\text { Diagnostic } \\
\text { Items }\end{array}$ & Nonwords \\
Fourth-year medical students & $908(34)$ & $683(20)$ & $747(16)$ \\
Physicians & $748(39)$ & $609(23)$ & $731(19)$
\end{tabular}

signs and symptoms. Inferences that were generated by at least $70 \%$ of the participants were selected as target items in the experiment. ${ }^{20}$ This approach ensures to a large extent that participants have sufficient prior knowledge to produce a particular inference and avoids the methodological danger of experimenters generating their own target items, assuming that readers also have the knowledge to generate such inferences. ${ }^{18}$ Although physicians and students generated many types of inferences, as has been outlined above, in our study we focused only on biomedical and diagnostic inferences.

Finally, the third prong consisted of a lexical decision task to focus on the differential role these types of inferences might have in the clinical reasoning processes of physicians and students. That is, what role does biomedical knowledge on the one hand, and diagnostic knowledge on the other, play in the physicians' and students' clinical reasoning processes? To explore this question, we used 60 short texts that were each followed by one of the 60 target items: 20 diagnostic items (i.e., clinical inferences that encapsulate the previously seen signs and symptoms, for instance, infarction); 20 biomedical items (i.e., items that referred to the underlying pathophysiology of one or more of the provided signs and symptoms, for instance, necrosis), and 20 nonwords. Participants had to decide as fast and as accurately as possible whether the presented item was a word or a nonword. It is important to note that we promoted semantic processing by using pseudohomophones as nonword stimuli (a pseudohomophone is a nonword that sounds like an existing word, for example "critilcosis" or "brane"), making the word/nonword decision more difficult. ${ }^{21}$ It was necessary to use pseudohomophones as nonwords because the participants could otherwise have used a simple strategy of making a decision based on the appearance of a word rather than by its meaning. The correct response to both diagnostic and biomedical targets was "yes."

\section{Procedure}

We instructed participants to carefully study short texts on a computer screen consisting of clinical signs and symptoms with the aim to determine the correct diagnosis. They were told that their performance on the decision task that followed each text would only profit considerably if they had carefully studied the texts. After 15 seconds, the text disappeared and a row of asterisks appeared in the center of the screen. The asterisks were replaced after 500 milliseconds by a target item, centered on the screen, which remained visible until the participant made a response by pressing keys on the computer keyboard. Participants had to decide as fast and as accurately as possible whether the presented item was a word or a nonword, by pressing the '?!' key for yes and ' $z$ ' key for no. Once a response was made, the target disappeared and the next text of signs and symptoms was presented. The presentation order of the texts was randomized for each participant. To ensure familiarity with the experimental procedure, five practice trials preceded the experimental trials. We tested all participants individually in a session that lasted approximately 30 minutes.

\section{Analysis}

For each participant, we collapsed the data to obtain a mean response time and a mean error rate for all levels of item type. We analyzed the data using a 2 (expertise level: fourth-year students and family physicians) $\times 3$ (item type: diagnostic items, biomedical items, and nonwords) mixed-design analysis of variance, with expertise level as the between-participants factor, and item type as the within-participants factor. Planned comparisons were made on the effects of item type. Effects were considered significant if the $p$ value was less than .05 .

\section{Results}

\section{Response times}

The mean response times of the physicians and medical students for the diagnostic items, biomedical items, and nonwords are shown in Table 1. Analysis of variance of the mean response times indicated a significant main effect of expertise level $\left(\mathrm{F}_{1,33}=7.92\right.$, mean squared error $[\mathrm{MSE}]=22766.34, p<$ $.01, \eta^{2}=.19$ ), a significant main effect of item type $\left(\mathrm{F}_{2,66}=43.73\right.$, MSE $=6493.31$, $p<.001, \eta^{2}=.57$ ), and a significant interaction between expertise level and item type $\left(\mathrm{F}_{2,66}=6.91\right.$, MSE $=6493.31$, $\left.p<.01, \eta^{2}=.17\right)$.

The planned comparison $t$ tests showed that physicians were significantly faster than were the medical students in judging the diagnostic items $(t[33]=2.51, p<$ $.05)$ and biomedical items $(t[33]=3.11$, $p<.01)$. We found no significant differences between students and physicians judging the nonwords. The item type comparisons within expertise level showed that physicians judged diagnostic items faster than biomedical items $(t[14]=7.26, p<.001)$ and nonwords $(t(14)=6.99, p<.001)$, but we found no significant difference between biomedical items and nonwords $(t[14]=.56, p=.58)$. Medical students judged diagnostic items significantly faster than biomedical items $(t[19]=$ $10.29, p<.001)$ and nonwords $(t[19]=$ $4.32, p<.001$ ), but they also judged nonwords significantly faster than biomedical items $(t[19]=2.51, p<.05)$.

\section{Error rates}

Table 2 shows the mean error rates (in percentages) of the physicians and students for both item types and nonwords. The analysis indicated no significant main effect of expertise level $(\mathrm{F}<1)$, but there was a borderline significant main effect of item type $\left(\mathrm{F}_{2,66}=2.98, \mathrm{MSE}=.56, p=.058\right.$, $\eta^{2}=.08$ ). Participants made slightly more mistakes judging the biomedical items than they did judging both other item types. Finally, we found no 
Table 2

Participants' Mean Error Rates in Percentages and Standard Errors, by Expertise Level and Item Type, from a Study on the Role of Biomedical Knowledge in Clinical Reasoning, Maastricht University Medical School, The Netherlands, 2002

\begin{tabular}{|c|c|c|c|}
\hline \multirow[b]{2}{*}{ Participant's expertise level } & \multicolumn{3}{|c|}{ Mean error rates (SE) } \\
\hline & $\begin{array}{r}\text { Biomedical } \\
\text { Items }\end{array}$ & $\begin{array}{r}\text { Diagnostic } \\
\text { Items }\end{array}$ & Nonwords \\
\hline Fourth-year medical students & $.70(.28)$ & $.30(.13)$ & $.20(.11)$ \\
\hline Physicians & $.53(.32)$ & $.27(.15)$ & $.20(.12)$ \\
\hline
\end{tabular}

significant interaction between expertise level and item type $(\mathrm{F}<1)$. It is important to note that physicians and students alike made hardly any mistakes, which validates the procedure of item selection.

The planned comparison $t$ tests showed no significant differences between family physicians and students. The item type comparisons within expertise level showed that physicians judged nonwords more accurately than biomedical items $(t[14]=2.65, p<.05)$. All other comparisons for the physicians were nonsigificant. The planned comparison $t$ tests showed no significant differences between item types for the medical students.

\section{Discussion}

In our study, we investigated the nature of inferences made by students and experienced family physicians using a lexical decision task. Participants were required to study a short clinical text that contained signs and symptoms associated with a particular disease. Based on knowledge encapsulation, we predicted that experienced physicians, in contrast to medical students, would not have a problem recognizing familiar constellations of signs and symptoms and hence would infer the associated diagnoses. Furthermore, we predicted that medical students would generate, as a result of their lack of clinical experience, primarily biomedical inferences in order to understand the signs and symptoms. Finally, we predicted that physicians would also outperform medical students on the biomedical items because the physician's biomedical knowledge is not only larger it is also better organized (i.e., encapsulated in clinical knowledge) than the student's biomedical knowledge. ${ }^{3-8}$

Our results are in accordance with knowledge encapsulation in that physicians judged diagnostic and biomedical items faster than did medical students. Furthermore, physicians needed less time judging diagnostic items than biomedical items. These findings seem to support the important assumption of knowledge encapsulation that biomedical knowledge still plays an important role in the physician's clinical reasoning. Moreover, the finding that physicians were even faster judging diagnostic items than biomedical items could be interpreted as evidence that diagnostic knowledge incorporates biomedical knowledge and has therefore an even more prominent role in the physician's clinical reasoning.

Our study also sheds a different light on previous studies that have used a traditional clinical case paradigm. These studies have found that experienced physicians hardly used biomedical knowledge in their recall or pathophysiological explanations of a clinical case. ${ }^{3-8}$ These findings have led to the assumption that biomedical knowledge is not involved during routine clinical case processing of experienced physicians. That is, experienced physicians immediately recognize the familiar constellations of signs and symptoms and infer the associated diagnosis without activating biomedical knowledge. ${ }^{7}$ However, our study shows that although recall protocols do not contain many explicit references to biomedical knowledge, this type of knowledge is still involved in the physicians' clinical reasoning. In other words, biomedical knowledge will become activated while processing the described signs and symptoms, although not as highly activated as diagnostic knowledge. In line with knowledge encapsulation, for an experienced physician, each sign and symptom described in the text refers to (i.e., activates) the diagnostic target item, but each sign and symptom does not necessarily refer to the selected biomedical target. So, more information in the text points to a particular diagnostic target item than to a particular biomedical target item and hence diagnostic items are more highly activated than biomedical items.

However, our finding that medical students judged diagnostic items and nonwords faster than biomedical items is more difficult to explain in light of knowledge encapsulation. We hypothesized that most students would have considerable difficulty generating an accurate diagnosis, leading to slower responses to diagnostic targets than to biomedical targets. Our findings could be interpreted by assuming that students only activated their diagnostic knowledge while reading our short texts and did not think about the biomedical targets until biomedical targets were presented. Perhaps the texts we used were not difficult enough for these advanced students. Moreover, these students were enrolled in a problem-based learning curriculum that introduces clinical aspects of disease early in the students' training. Consequently, students did not have to use their biomedical knowledge extensively in order to establish a meaningful relation between the described signs and symptoms and processed them in essentially the same way as did the family physicians. On the other hand, it is important to note that response times indicate that the physician's medical knowledge (i.e., biomedical and clinical knowledge) is better organized and integrated than that of students. That is, physicians were clearly faster than were students on biomedical and diagnostic targets and, moreover, evaluated these medical targets significantly faster than nonwords.

It could be argued that this pattern of results (i.e., physicians are faster than students on both item types) is a consequence of differences between students and physicians in exposure to these items. So, physicians might outperform students on diagnostic and biomedical targets simply because they have encountered these targets more often throughout their career. This frequency effect will result in faster response times to these items - even without the preceding text. Although this alternative explanation is almost intuitively plausible, it cannot 
completely account for our data. First of all, it is important to note that the students in our study were already in their fourth year of medical training and hence very familiar with the target items used on our study. Furthermore, we selected target items based on their familiarity for both students and physicians by using the three-pronged approach. Secondly, frequency of encounter does not necessarily lead to improved performance. For instance, a person who is 50 years of age has encountered in his or her lifetime many words more often than a person who is only 20 years of age. Still, these many extra years of practice do not automatically result in a decrease in response time on a lexical decision task. ${ }^{22}$ As a matter of fact, lexical decision response times increase considerably with age. ${ }^{23}$

Interestingly, our findings are in line with a recent study by Rikers and colleagues. ${ }^{24}$ They also asked family physicians and advanced students to study short texts associated with a particular disease, but in contrast to our study, their participants were asked to judge the relatedness of targets items that followed each text. Targets were biomedical or diagnostic items that were either related or unrelated to the text. Similar to our study, physicians judged related biomedical and diagnostic items faster than did students. Furthermore, physicians and students judged diagnostic targets faster than biomedical targets. In line with our study, these findings indicate that both physicians and students were able to generate diagnostic and biomedical inferences, but physicians can generate them faster.

As we expected, the error rates in our study were low and did not significantly differ between physicians and students. As has been outlined before, the target items used during the decision task were selected based on their familiarity for students and physicians and consequently participants hardly made mistakes. With this procedure the experimenter can safely argue that the participants have a sufficient amount of background knowledge to produce a particular inference. Otherwise there is a methodological hazard in experimenters generating their own inferences and presuming that participants have the knowledge to generate such inferences. ${ }^{16,18,19}$
In sum, our findings indicate that biomedical knowledge has not lost its function in the physician's clinical reasoning. Furthermore, our findings indicate that the main difference between experienced family physicians and advanced medical students is not the ability to generate diagnostic or biomedical inferences, but the role these types of inferences play in clinical reasoning. That is, diagnostic and biomedical inferences are easily generated by physicians through their encapsulated knowledge and are the building blocks of their clinical reasoning. However, further research is needed to focus more specifically on why the advanced students in our study also evaluated diagnostic items faster than biomedical items, although most theories on expertise development assume that biomedical knowledge plays a more prominent role in the student's clinical case representation than in the physician's case representation.

\section{References}

1 Patel VL, Evans DA, Groen GJ. Biomedical knowledge in clinical reasoning. In: Evans DA, Patel VL (eds). Cognitive Science in Medicine: Biomedical Modeling. Cambridge: MIT Press, 1989:53-112.

2 Patel VL, Groen GJ, Arocha JF. Medical expertise as a function of task difficulty. Mem Cogn. 1990;18:394-406.

3 Rikers RMJP, Boshuizen HPA, Schmidt HG. Knowledge encapsulation and the intermediate effect. Contemp Educ Psychol. 2000;25:150-66.

4 Rikers RMJP, Schmidt HG, Boshuizen HPA. On the constraints of encapsulated knowledge: clinical case representations by medical experts and subexperts. Cogn Instr. 2002;20:27-46.

5 Rikers RMJP, Schmidt HG, Boshuizen HPA, Linssen GCM, Wesseling G, Paas FGWC. The robustness of medical expertise: clinical case processing by medical experts and subexperts. Am J Psychol. 2002;115:609-29.

6 Schmidt HG, Boshuizen HPA. On the origin of intermediate effects in clinical case recall. Mem Cogn. 1993;21:338-51.

7 de Bruin ABH, Schmidt HG, Rikers RMJP. The role of basic science knowledge and clinical knowledge in diagnostic reasoning: a structural equation modeling approach. Acad Med. 2005:80;765-73.

8 Schmidt HG, Boshuizen HPA, Hobus PPM. Transitory stages in the development of medical expertise: the "intermediate effect" in clinical case representation studies. In: Proceedings of the Cognitive Science Society Meeting. Hillsdale, NJ: Lawrence Erlbaum Associates, 1988:139-45.
9 Blessing SB, Anderson JR. How people learn to skip steps. J Exp Psychol Learn Mem Cogn. 1996;22:576-98.

10 Koedinger KR, Anderson JR. Abstract planning and perceptual chunks: elements of expertise in geometry. Cogn Sci. 1990;14:511-50.

11 Patel VL, Ramoni MF. Cognitive models of directional inference in expert medical reasoning. In: Feltovich PJ, Ford KM, Hoffman RR (eds). Expertise in Context: Human and Machine. Cambridge: MIT Press, 1998:67-99.

12 Patel VL, Kaufman DR. Clinical reasoning and biomedical knowledge: implications for teaching. In: Higgs J, Jones M (eds.) Clinical Reasoning in the Health Professions. Oxford: Butterworth Heinemann, 1995:117-28.

13 Lesgold AM . Acquiring expertise. In: Anderson JR, Kosslyn SM (eds). Tutorials in Learning and Memory: Essays in Honor of Gordon Bower. San Francisco: Freeman, 1984:31-60.

14 Lesgold AM, Rubinson H, Feltovich PJ, Glaser R, Klopfer D, Wang Y. Expertise in a complex skill: diagnosing X-ray pictures. In: Chi MTH, R. Glaser R, Farr M (eds). The Nature of Expertise. Hillsdale, NJ: Erlbaum, 1988:311-42.

15 Graesser AC, Singer M, Trabasso T. Constructing inferences during narrative text comprehension. Psychol Rev. 1994;101:371-95.

16 Graesser AC, Wiemer-Hastings K. Situation models and concepts in story comprehension. In: Goldman SR, Graesser AC, van den Broek P (eds). Narrative Comprehension, Causality, and Coherence: Essays in Honor of Tom Trabasso. Mahwah, NJ: Erlbaum, 1999:77-92.

17 Margolis MD. Johns Hopkins Symptoms and Remedies, Revised Edition. New York: Times Books, 1999.

18 Magliano JP, Graesser AC. A three-pronged method for studying inference generation in literary texts. Poetics. 1991;20:193-232.

19 Suh S, Trabasso T. Inferences during reading: converging evidence from discourse analysis, talk-aloud protocols and recognition priming. J Mem Lang. 1993;32:27-30.

20 Leon JA, Perez O. The influence of prior knowledge on the time course of clinical diagnosis inferences: a comparison of experts and novices. Discourse Process. 2001;31:187-213.

21 Joordens S, Becker S. The long and short of semantic priming effects in lexical decision. J Exp Psychol Learn Mem Cogn. 1997;23:1083-105.

22 Ratcliff R, Thapar A, Gomez P, McKoon G. A diffusion model analysis of the effects of aging in the lexical-decision task. Psychol Aging. 2004;19:278-89.

23 Allen PA, Madden DJ, Crozier LC. Adult age differences in letter-level and word-level processing. Psychol Aging. 1991;6:261-71.

24 Rikers RMJP, Schmidt HG, Moulaert V. Biomedical knowledge: encapsulated or two worlds apart? Appl Cogn Psychol. 2005;19: $223-31$. 